\title{
Positive periodic solutions of second-order difference equations with weak singularities
}

\author{
Ruyun $\mathrm{Ma}^{*}$ and Yanqiong Lu
}

* Correspondence: mary@nwnu. edu.cn

Department of Mathematics, Northwest Normal University, Lanzhou 730070, People's Republic of China

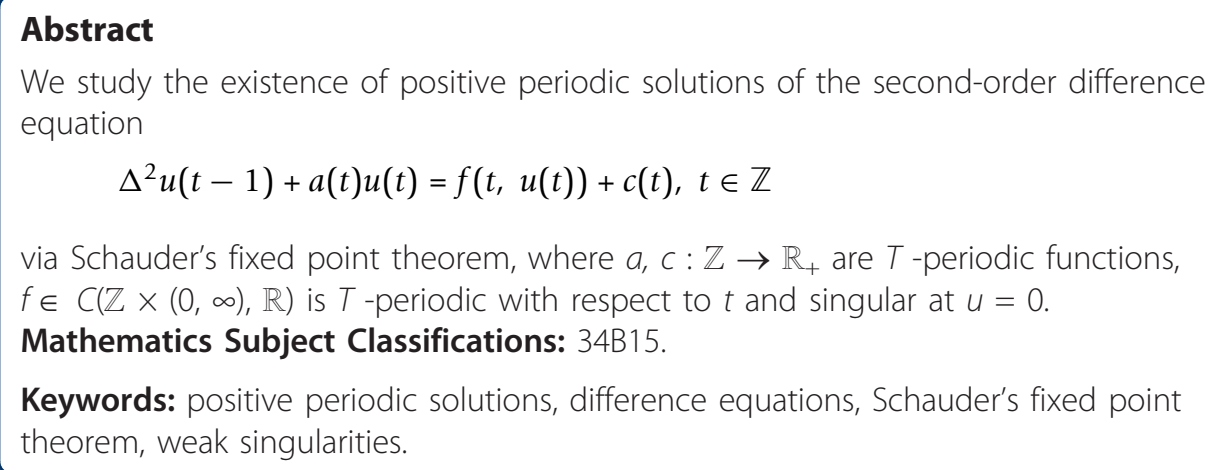

We study the existence of positive periodic solutions of the second-order difference equation

$$
\Delta^{2} u(t-1)+a(t) u(t)=f(t, u(t))+c(t), t \in \mathbb{Z}
$$

via Schauder's fixed point theorem, where $a, c: \mathbb{Z} \rightarrow \mathbb{R}_{+}$are $T$-periodic functions, $f \in C(\mathbb{Z} \times(0, \infty), \mathbb{R})$ is $T$-periodic with respect to $t$ and singular at $u=0$.

Mathematics Subject Classifications: 34B15.

Keywords: positive periodic solutions, difference equations, Schauder's fixed point theorem, weak singularities.

\section{Introduction and the main results}

Let $\mathbb{Z}$ denote the integer set, for $a, b \in \mathbb{Z}$ with $a<b,[a, b]_{\mathbb{Z}}:=\{a, a+1, \ldots, b\}$ and $\mathbb{R}_{+}:=$ $[0 ; \infty)$. In this article, we are concerned with the existence of positive periodic solutions of the second-order difference equation

$$
\Delta^{2} u(t-1)+a(t) u(t)=f(t, u(t))+c(t), \quad t \in \mathbb{Z},
$$

where $a, c: \mathbb{Z} \rightarrow \mathbb{R}_{+}$are $T$-periodic functions, $f \in C(\mathbb{Z} \times(0, \infty), \mathbb{R})$ is $T$-periodic with respect to $t$ and singular at $u=0$.

Positive periodic solutions of second-order difference equations have been studied by many authors, see [1-6]. However, in these therein, the nonlinearities are nonsingular, what would happen if the nonlinearity term is singular? It is of interest to note here that singular boundary value problems in the continuous case have been studied in great detail in the literature [7-20]. In 1987, Lazer and Solimini [7] firstly investigated the existence of the positive periodic solutions of the problem

$$
u^{\prime \prime}=\frac{1}{u^{\lambda}}+c(t)
$$

where $c \in C(\mathbb{R}, \mathbb{R})$ is $T$-periodic. They proved that for $\lambda \geq 1$ (called strong force condition in a terminology first introduced by Gordon $[8,9])$, a necessary and sufficient condition for the existence of a positive periodic solution of (1.2) is that the mean value of $c$ is negative,

(c) $2012 \mathrm{Ma}$ and Lu; licensee Springer. This is an Open Access article distributed under the terms of the Creative Commons Attribution License (http://creativecommons.org/licenses/by/2.0), which permits unrestricted use, distribution, and reproduction in any medium, provided the original work is properly cited. 


$$
\bar{c}:=\frac{1}{T} \int_{0}^{T} c(t) d t<0
$$

Moreover, if $0<\lambda<1$ (weak force condition) they found examples of functions $c$ with negative mean values and such that periodic solutions do not exist. Subsequently, many authors studied the existence of positive solutions of the problem

$$
u^{\prime \prime}+a(t) u=f(t, u)+c(t),
$$

where $a \in L^{1}\left(\mathbb{R} / T \mathbb{Z} ; \mathbb{R}_{+}\right), c \in L^{1}(\mathbb{R} / T \mathbb{Z} ; \mathbb{R}), f \in \operatorname{Car}(\mathbb{R} / T \mathbb{Z} \times(0, \infty), \mathbb{R})$ and is singular at $u=0$, see [7-20]. The first existence result with weak force condition appears in Rachunková et al. [16]. Since then, the Equation (1.3) with $f$ has weak singularities has been studied by several authors, see Torres [17,18], Franco and Webb [19], Chu and Li [20].

Recently, Torres [18] showed how a weak singularity can play an important role if Schauder's fixed point theorem is chosen in the proof of the existence of positive periodic solution for (1.3). For convenience, for a given function $\xi \in L^{\infty}[0, T]$, we denote the essential supremum and infimum of $\xi$ by $\xi^{*}$ and $\xi_{*}$, respectively. We write $\xi>0$ if $\xi \geq 0$ for a.e. $t \in[0, T]$ and it is positive in a set of positive measure. Under the assumption

(H1) The linear equation $u^{\prime \prime}+a(t) u=0$ is nonresonant and the corresponding Green's function

$$
G(t, s) \geq 0, \quad(t, s) \in[0, T] \times[0, T] .
$$

Torres showed the following three results

Theorem A. [[18], Theorem 1] Let (H1) hold and define

$$
\gamma(t)=\int_{0}^{T} G(t, s) c(s) d s .
$$

Assume that

(H2) there exist $b \in L^{1}(0, T)$ with $b>0$ and $\lambda>0$ such that

$$
0 \leq f(t, u) \leq \frac{b(t)}{u^{\lambda}}, \quad \text { for all } u>0, \quad \text { a.e. } t \in[0, T]
$$

If $\gamma$ * $>0$, then there exists a positive $T$-periodic solution of (1.3).

Theorem B. [[18], Theorem 2] Let (H1) hold. Assume that

(H3) there exist two functions $b, \hat{b} \in L^{1}(0, T)$ with $\mathrm{b}, \hat{b} \succ 0$ and a constant $\lambda \in(0,1)$ such that

$$
0 \leq \frac{\hat{b}(t)}{u^{\lambda}} \leq f(t, u) \leq \frac{b(t)}{u^{\lambda}}, u \in(0, \infty), \text { a.e. } t \in[0, T]
$$

If $\gamma_{*}=0$. Then (1.3) has a positive $T$-periodic solution.

Theorem C. [[18], Theorem 4] Let (H1) and (H3) hold. Let

$$
\hat{\beta}_{*}=\min _{t \in[0, T]}\left(\int_{0}^{T} G(t, s) \hat{b}(s) d s\right), \quad \beta^{*}=\max _{t \in[0, T]}\left(\int_{0}^{T} G(t, s) b(s) d s\right) .
$$


If $\gamma^{*} \leq 0$ and

$$
\gamma_{*} \geq\left(\frac{\hat{\beta}_{*}}{\left(\beta^{*}\right)^{\lambda}} \lambda^{2}\right)^{\frac{1}{1-\lambda^{2}}}\left(1-\frac{1}{\lambda^{2}}\right) .
$$

Then (1.3) has a positive $T$-periodic solution.

However, the discrete analogue of (1.3) has received almost no attention. In this article, we will discuss in detail the singular discrete problem (1.1) with our goal being to fill the above stated gap in the literature. For other results on the existence of positive solution for the other singular discrete boundary value problem, see [21-24] and their references. From now on, for a given function $\xi \in l^{\infty}(0, \infty)$, we denote the essential supremum and infimum of $\xi$ by $\xi^{*}$ and $\xi_{*}$, respectively. We write $\xi>0$ if $\xi \geq 0$ for $t[0$, $T]_{\mathrm{Z}}$ and it is positive in a set of positive measure.

Assume that

(A1) The linear equation $\Delta^{2} u(t-1)+a(t) u(t)=0$ is nonresonant and the corresponding Green's function

$$
G(t, s) \geq 0, \quad(t, s) \in[0, T]_{\mathbb{Z}} \times[0, T]_{\mathbb{Z}} .
$$

(A2) There exist $b, e:[1, T]_{\mathrm{Z}} \rightarrow \mathbb{R}_{+}$with $b, e>0, \alpha, \beta \in(0, \infty), m \leq 1 \leq M$, such that

$$
0 \leq f(t, u) \leq \frac{b(t)}{u^{\alpha}}, \quad u \in(M, \infty), \quad t \in[1, T]_{\mathbb{Z}}
$$

and

$$
0 \leq f(t, u) \leq \frac{e(t)}{u^{\beta}}, \quad u \in(0, m), \quad t \in[1, T]_{\mathbb{Z}} .
$$

(A3) There exist $b_{1}, b_{2}, e:[1, T]_{Z} \rightarrow \mathbb{R}_{+}$with $b_{1}, b_{2}, e>0, \alpha, \beta, \mu, v \in(0,1)$, such that

$$
0 \leq \frac{b_{1}(t)}{u^{\alpha}} \leq f(t, u) \leq \frac{b_{2}(t)}{u^{\beta}}, \quad u \in[1, \infty), t \in[1, T]_{\mathbb{Z}}
$$

and

$$
0 \leq \frac{b_{1}(t)}{u^{\mu}} \leq f(t, u) \leq \frac{e(t)}{u^{v}}, \quad u \in[0,1), \quad t \in[1, T]_{\mathbb{Z}} .
$$

To prove the main results, we will use the following notations.

$$
\begin{aligned}
& \gamma(t):=\sum_{s=1}^{T} G(t, s) c(s), \quad E(t):=\sum_{s=1}^{T} G(t, s) e(s) \\
& B(t):=\sum_{s=1}^{T} G(t, s) b(s), \quad B_{i}(t):=\sum_{s=1}^{T} G(t, s) b_{i}(s), \quad i=1,2 \\
& \rho^{*}:=E^{*}+B_{2}^{*}, \quad \sigma:=\max \{\mu, \alpha\}, \quad \delta:=\max \{v, \beta\} .
\end{aligned}
$$

Our main results are the following 
Theorem 1.1. Let (A1) and (A2) hold. If $\gamma_{*}>0$. Then (1.1) has a positive $T$-periodic solution.

Theorem 1.2. Let (A1) and (A3) hold. If $\gamma_{*}=0$. Then (1.1) has a positive $T$-periodic solution.

Theorem 1.3. Let (A1) and (A3) hold. Assume that

$$
\rho^{*}>\max \left\{\left(\delta \sigma B_{1_{*}}\right)^{\delta},\left(\delta \sigma B_{1_{*}}\right) \frac{1}{\sigma}\right\} .
$$

If $\gamma^{*} \leq 0$ and

$$
\gamma_{*} \geq\left[\frac{B_{1_{*}}}{\left(\rho^{*}\right)^{\sigma}} \delta \sigma\right]^{\frac{1}{1-\delta \sigma}}\left(1-\frac{1}{\delta \sigma}\right) .
$$

Then (1.1) has a positive $T$-periodic solution.

Remark 1.1. Let us consider the function

$$
f_{0}(t, u)= \begin{cases}\frac{1}{u^{\epsilon}}, & u \in[1, \infty), \\ \frac{1}{u^{\eta}}, & u \in(0,1),\end{cases}
$$

where $\varepsilon, \eta>0$. Obviously, $f_{0}$ satisfies (A2) with $M=m=1, b(t)=e(t) \equiv 1$. However, it is fail to satisfy (H2) since it can not be bounded by a single function $\frac{h(t)}{u^{\gamma}}$ for any $\gamma$ $\in(0, \infty)$ and any $h>0$. $\square$

Remark 1.2. If $\varepsilon, \eta \in(0,1)$, then the function $f_{0}$ defined by (1.6) satisfies (A3) with $v$ $=\mu=\eta, \alpha=\beta=\varepsilon$, and $b_{1}(t) \equiv b_{2}(t) \equiv e(t) \equiv 1$. However, it is fail to satisfy (H3).

\section{Proof of Theorem 1.1}

Let

$$
X:=\{u: \mathbb{Z} \rightarrow \mathbb{R} \mid u(t)=u(t+T)\}
$$

under the norm $\|u\|=\max _{t \in[1, T]_{\mathbb{Z}}}|u(t)|$. Then $(X,\|\cdot\|)$ is a Banach space.

A $T$-periodic solution of (1.1) is just a fixed point of the completely continuous map $A: X \rightarrow X$ defined as

$$
(A u)(t):=\sum_{s=1}^{T} G(t, s)[f(s, u(s))+c(s)]=\sum_{s=1}^{T} G(t, s) f(s, u(s))+\gamma(t) .
$$

By Schauder's fixed point theorem, the proof is finished if we prove that $A$ maps the closed convex set defined as

$$
K=\left\{u \in X: r \leq u(t) \leq R, \quad \text { for all } t \in[0, T]_{\mathbb{Z}}\right\}
$$

into itself, where $R>r>0$ are positive constants to be fixed properly.

For given $u \in K$, let us denote

$$
\begin{aligned}
& I_{1}:=\left\{t \in[0, T]_{\mathbb{Z}} \mid r \leq u(t)<m\right\}, \\
& I_{2}:=\left\{t \in[0, T]_{\mathbb{Z}} \mid R \geq u(t)>M\right\},
\end{aligned}
$$

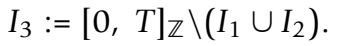


Given $u \in K$, by the nonnegative sign of $G$ and $f$, we have

$$
\begin{aligned}
(A u)(t)= & \sum_{s=1}^{T} G(t, s) f(s, u(s))+\gamma(t) \\
= & \sum_{s \in I_{1}} G(t, s) f(s, u(s))+\sum_{s \in I_{2}} G(t, s) f(s, u(s)) \\
& +\sum_{s \in I_{3}} G(t, s) f(s, u(s))+\gamma(t) \\
\geq & \gamma(t) \geq \gamma_{*}=: r .
\end{aligned}
$$

Let

$$
\Lambda:=\sup \left\{\max _{t \in[0, T]_{\mathbb{Z}}} \sum_{s=1}^{T} G(t, s) f(s, u(s)) \mid m \leq u(s) \leq M\right\} .
$$

Then, it follows from the continuity of $f$ that $\Lambda<\infty$, and consequently, for every $u \in$ $K$,

$$
\begin{aligned}
(A u)(t)= & \sum_{s=1}^{T} G(t, s) f(s, u(s))+\gamma(t) \\
= & \sum_{s \in I_{1}} G(t, s) f(s, u(s))+\sum_{s \in I_{2}} G(t, s) f(s, u(s)) \\
& +\sum_{s \in I_{3}} G(t, s) f(s, u(s))+\gamma(t) \\
\leq & \sum_{s \in I_{1}} G(t, s) \frac{e(s)}{u^{\beta}}+\sum_{s \in I_{2}} G(t, s) \frac{b(s)}{u^{\alpha}}+\Lambda+\gamma^{*} \\
\leq & \sum_{s=1}^{T} G(t, s) \frac{e(s)}{u^{\beta}}+\sum_{s \in I_{2}} G(t, s) b(s)+\Lambda+\gamma^{*} \\
\leq & \sum_{s=1}^{T} G(t, s) \frac{e(s)}{r^{\beta}}+\sum_{s=1}^{T} G(t, s) b(s)+\Lambda+\gamma^{*} \\
\leq & \frac{E^{*}}{r^{\beta}}+\left(B^{*}+\Lambda+\gamma^{*}\right) \\
< & \frac{E^{*}}{r^{\beta}}+\left(B^{*}+\Lambda+\gamma^{*}\right)=: R .
\end{aligned}
$$

Therefore, $A(K) \subset K$ if $r=\gamma_{*}$ and $R=\frac{E^{*}}{r^{\beta}}+\left(B^{*}+\Lambda+\gamma^{*}\right)$, and the proof is finished. $\square$

\section{Proof of Theorem 1.2}

We follow the same strategy and notations as in the proof of Theorem 1.1. Define a closed convex set

$$
K=\left\{u \in X: r \leq u(t) \leq R, \quad \text { for all } t \in[0, T]_{\mathbb{Z}}, R>1\right\} .
$$

By a direct application of Schauder's fixed point theorem, the proof is finished if we prove that $A$ maps the closed convex set $K$ into itself, where $R$ and $r$ are positive constants to be fixed properly and they should satisfy $R>r>0$ and $R>1$. 
For given $u \in K$, let us denote

$$
\begin{aligned}
& J_{1}:=\left\{t \in[0, T]_{\mathbb{Z}} \mid r \leq u(t)<1\right\}, \\
& J_{2}:=\left\{t \in[0, T]_{\mathbb{Z}} \mid R \geq u(t) \geq 1\right\} .
\end{aligned}
$$

Then for given $u \in K$, by the nonnegative sign of $G$ and $f$, it follows that

$$
\begin{aligned}
(A u)(t) & =\sum_{s=1}^{T} G(t, s) f(s, u(s))+\gamma(t) \\
& =\sum_{s \in J_{1}}^{T} G(t, s) f(s, u(s))+\sum_{s \in J_{2}} G(t, s) f(s, u(s))+\gamma(t) \\
& \leq \sum_{s \in J_{1}} G(t, s) \frac{e(s)}{u^{v}}+\sum_{s \in J_{2}} G(t, s) \frac{b_{2}(s)}{u^{\beta}}+\gamma^{*} \\
& \leq \sum_{s=1}^{T} G(t, s) \frac{e(s)}{r^{v}}+\sum_{s \in J_{2}} G(t, s) b_{2}(s)+\gamma^{*} \\
& \leq \sum_{s=1}^{T} G(t, s) \frac{e(s)}{r^{v}}+\sum_{s=1}^{T} G(t, s) b_{2}(s)+\gamma^{*} \\
& \leq \frac{E^{*}}{r^{v}}+\left(B_{2}^{*}+\gamma^{*}\right),
\end{aligned}
$$

On the other hand, for every $u \in K$,

$$
\begin{aligned}
(A u)(t) & =\sum_{s=1}^{T} G(t, s) f(s, u(s))+\gamma(t) \\
& =\sum_{s \in J_{1}} G(t, s) f(s, u(s))+\sum_{s \in J_{2}} G(t, s) f(s, u(s))+\gamma(t) \\
& \geq \sum_{s \in J_{1}} G(t, s) \frac{b_{1}(s)}{u^{\mu}}+\sum_{s \in J_{2}} G(t, s) \frac{b_{1}(s)}{u^{\alpha}}+\gamma_{*} \\
& \geq \sum_{s \in J_{1}} G(t, s) \frac{b_{1}(s)}{R^{\mu}}+\sum_{s \in J_{2}} G(t, s) \frac{b_{1}(s)}{R^{\alpha}} \\
& \geq \sum_{s \in J_{1}} G(t, s) \frac{b_{1}(s)}{R^{\sigma}}+\sum_{s \in J_{2}} G(t, s) \frac{b_{1}(s)}{R^{\sigma}} \\
& \geq \sum_{s=1}^{T} G(t, s) \frac{b_{1}(s)}{R^{\sigma}} \\
& \geq \frac{B_{1_{*}}}{R^{\sigma}} .
\end{aligned}
$$

Thus $A u \in K$ if $r, R$ are chosen so that

$$
\frac{B_{1 *}}{R^{\sigma}} \geq r, \quad \frac{E^{*}}{r^{v}}+\left(B_{2}^{*}+\gamma^{*}\right) \leq R .
$$

Note that $B_{1^{*}}, E^{*}>0$ and taking $R=\frac{1}{r}$, it is sufficient to find $R>1$ such that

$$
B_{1 *} R^{1-\sigma} \geq 1, \quad E^{*} R^{v}+\left(B_{2}^{*}+\gamma^{*}\right) \leq R,
$$

and these inequalities hold for $R$ big enough because $\sigma<1$ and $v<1$. $\square$ 
Remark 3.1. It is worth remarking that Theorem 1.2 is also valid for the special case that $c(t) \equiv 0$, which implies that $\gamma_{*}=0$.

\section{Proof of Theorem 1.3}

Define a closed convex set

$$
K=\left\{u \in X: r \leq u(t) \leq R, \quad \text { for all } t \in[0, T]_{\mathbb{Z}}, \quad 0<r<1<R\right\} .
$$

By a direct application of Schauder's fixed point theorem, the proof is finished if we prove that $A$ maps the closed convex set $K$ into itself, where $R$ and $r$ are positive constants to be fixed properly and they should satisfy $R>1>r>0$.

Recall that $\delta=\max \{v, \beta\}$ and $r<1$, for given $u \in K$,

$$
\begin{aligned}
(A u)(t) & =\sum_{s=1}^{T} G(t, s) f(s, u(s))+\gamma(t) \\
& =\sum_{s \in J_{1}} G(t, s) f(s, u(s))+\sum_{s \in J_{2}} G(t, s) f(s, u(s))+\gamma(t) \\
& \leq \sum_{s \in J_{1}} G(t, s) \frac{e(s)}{u^{v}}+\sum_{s \in J_{2}} G(t, s) \frac{b_{2}(s)}{u^{\beta}}+\gamma^{*} \\
& \leq \sum_{s \in J_{1}} G(t, s) \frac{e(s)}{r^{\nu}}+\sum_{s \in J_{2}} G(t, s) \frac{b_{2}(s)}{r^{\beta}} \\
& \leq \sum_{s=1}^{T} G(t, s) \frac{e(s)}{r^{\delta}}+\sum_{s=1}^{T} G(t, s) \frac{b_{2}(s)}{r^{\delta}} \\
& \leq \frac{\rho^{*}}{r^{\delta}}
\end{aligned}
$$

where $J_{i}(i=1,2)$ is defined as in Section 3 and $\rho^{*}=E^{*}+B_{2}^{*}$.

On the other hand, since $\sigma=\max \{\mu, \alpha\}$ and $R>1$, for every $u \in K$,

$$
\begin{aligned}
(A u)(t) & =\sum_{s=1}^{T} G(t, s) f(s, u(s))+\gamma(t) \\
& =\sum_{s \in J_{1}} G(t, s) f(s, u(s))+\sum_{s \in J_{2}} G(t, s) f(s, u(s))+\gamma(t) \\
& \geq \sum_{s \in J_{1}} G(t, s) \frac{b_{1}(s)}{u^{\mu}}+\sum_{s \in J_{2}} G(t, s) \frac{b_{1}(s)}{u^{\alpha}}+\gamma_{*} \\
& \geq \sum_{s \in J_{1}} G(t, s) \frac{b_{1}(s)}{R^{\sigma}}+\sum_{s \in J_{2}} G(t, s) \frac{b_{1}(s)}{R^{\sigma}}+\gamma_{*} \\
& \geq \frac{B_{1 *}}{R^{\sigma}}+\gamma_{*} .
\end{aligned}
$$

In this case, to prove that $A(K) \subset K$ it is sufficient to find $r<R$ with $0<r<1<R$ such that

$$
\frac{B_{1 *}}{R^{\sigma}}+\gamma_{*} \geq r, \quad \frac{\rho^{*}}{r^{\delta}} \leq R
$$

If we fix $R=\frac{\rho^{*}}{r^{\delta}}$, then the first inequality holds if $r$ verifies

$$
\frac{B_{1 *}}{\left(\rho^{*}\right)^{\sigma}} r^{\sigma \delta}+\gamma_{*} \geq r
$$


or equivalently,

$$
\gamma_{*} \geq f(r):=r-\frac{B_{1 *}}{\left(\rho^{*}\right)^{\sigma}} r^{\sigma \delta} .
$$

The function $f(r)$ possesses a minimum in $r_{0}:=\left[\frac{B_{1 *}}{\left(\rho^{*}\right)^{\sigma}} \delta \sigma\right]^{\frac{1}{1-\delta \sigma}}$. Taking $r=r_{0}$, (1.4) implies that $r<1$. Then the first inequality in (4.1) holds if $\gamma_{*} \geq f\left(r_{0}\right)$, which is just condition (1.5). The second inequality in (4.1) holds directly by the choice of $R$, and it would remain to prove that $R=\frac{\rho^{*}}{r_{0}^{\delta}}>1$. To the end, it follows from (1.4) that

$$
\begin{aligned}
R & =\frac{\rho^{*}}{r_{0}^{\delta}}>\frac{\left(\delta \sigma B_{1 *}\right)^{\delta} \cdot\left(\rho^{*}\right)^{\frac{\delta \sigma}{1-\delta \sigma}}}{\left(\delta \sigma B_{1 *}\right)^{\frac{\delta}{1-\delta \sigma}}} \\
& >\frac{\left(\delta \sigma B_{1 *}\right)^{\delta} \cdot\left(\delta \sigma B 1_{*}\right)^{\frac{\delta^{2} \sigma}{1-\delta \sigma}}}{\left(\delta \sigma B_{1 *}\right)^{\frac{B_{1} \delta}{1-\delta \sigma}}} \\
& =1 .
\end{aligned}
$$

This completes the proof. $\square$

Remark 4.1. Note that the condition (1.4), which is stated as

$$
\rho^{*}>\max \left\{\left(\delta \sigma B_{1 *}\right)^{\delta},\left(\delta \sigma B_{1 *}\right)^{\frac{1}{\sigma}}\right\}
$$

is crucial to guarantee that $R>1>r_{0}$, and in the proof of Theorem 1.3 we require $R$ $>1>r_{0}$ because the exponents in inequalities of (A3) is different. However, in the special case that

$$
\lambda:=\alpha=\beta=\mu=\nu,
$$

if we define $\omega(t):=\max \left\{b_{2}(t), e(t)\right\}, t \in[0, T]_{Z}$, then the condition (1.4) is needn't because $R>r_{0}$ can be easily verified by

$$
b_{1}(t) \leq \omega(t), t \in[0, T]_{\mathbb{Z}} .
$$

$\square$

Example 4.1. Let us consider the second order periodic boundary value problem

$$
\begin{aligned}
& \Delta^{2} u(t-1)+4 \sin ^{2} \frac{\pi}{16} u=f(t, u)-c_{0}, \quad t \in[1,4]_{\mathbb{Z}} \\
& u(0)=u(4), \quad u(1)=u(5),
\end{aligned}
$$

where

$$
f(t, u)=\frac{5-t}{u^{\frac{1}{5}}}, \quad u \in(0, \infty), t \in[1,4]_{\mathbb{Z}}
$$

and $c_{0} \in\left(0, \frac{3 \cdot[8 \sqrt{10}]^{-4 / 3}}{((4+3 \sqrt{2}) \sqrt{2-\sqrt{2}}+2 \sqrt{2})^{1 / 3}}\right)$ is a constant. 
It is easy to check that (4.2) is equivalent to the operator equation

$$
u(t)=\sum_{s=1}^{4} G(t, s) f(s, u(s))+\sum_{s=1}^{4} G(t, s)\left(-c_{0}\right) d s=:(A u)(t), \quad t \in[0,4]_{\mathbb{Z}},
$$

here

$$
G(t, s)=\left\{\begin{array}{l}
\frac{1}{\sin \frac{\pi}{8}}\left[\sin \frac{\pi(t-s)}{8}+\sin \frac{\pi(4-t+s)}{8}\right], 0 \leq s \leq t \leq 4 \\
\frac{1}{\sin \frac{\pi}{8}}\left[\sin \frac{\pi(s-t)}{8}+\sin \frac{\pi(4-s+t)}{8}\right], 0 \leq t \leq s \leq 4
\end{array}\right.
$$

Clearly, $G(t, s)>0$ for all $(t, s) \in 0[4]_{Z} \times 0[4]_{Z}$.

Let

$$
\begin{aligned}
& b_{1}(t) \equiv 1, \quad b_{2}(t) \equiv 4, \quad e(t) \equiv 6, \\
& \alpha=v=\frac{1}{2}, \quad \beta=\frac{1}{6}, \quad \mu=\frac{1}{7},
\end{aligned}
$$

Then

$$
\sigma=\delta=\frac{1}{2}
$$

and

$$
\begin{aligned}
& 0<\frac{1}{u^{\frac{1}{2}}} \leq \frac{4-t}{u^{\frac{1}{5}}} \leq \frac{4}{u^{\frac{1}{6}}}, \quad u \in[1, \infty), \quad t \in[0, T]_{\mathbb{Z}} \\
& 0<\frac{1}{u^{\frac{1}{7}}} \leq \frac{4-t}{u^{\frac{1}{5}}} \leq \frac{6}{u^{\frac{1}{2}}}, \quad u \in[0,1), \quad t \in[0, T]_{\mathbb{Z} .}
\end{aligned}
$$

Thus, the condition (A3) is satisfied. By simple computations, we get

$$
\begin{aligned}
& B_{1}(t)=\sum_{s=1}^{4} G(t, s) \cdot \frac{1}{2}=\frac{(4+3 \sqrt{2}) \sqrt{2-\sqrt{2}}}{2}+\sqrt{2} ; \\
& B_{2}(t)=\sum_{s=1}^{4} G(t, s) \cdot 4=(16+12 \sqrt{2}) \sqrt{2-\sqrt{2}}+8 \sqrt{2} ; \\
& E(t)=\sum_{s=1}^{4} G(t, s) \cdot 6=(24+18 \sqrt{2}) \sqrt{2-\sqrt{2}}+12 \sqrt{2} ; \\
& B_{1 *}=B_{1}^{*}=\frac{(4+3 \sqrt{2}) \sqrt{2-\sqrt{2}}}{2}+\sqrt{2} ; \\
& B_{2 *}=B_{2}^{*}=(16+12 \sqrt{2}) \sqrt{2-\sqrt{2}}+8 \sqrt{2} \text {; } \\
& E_{*}=E^{*}=(24+18 \sqrt{2}) \sqrt{2-\sqrt{2}}+12 \sqrt{2} \text {; } \\
& \left(\delta \sigma B_{1 *}\right)^{\delta}=\left[\frac{(4+3 \sqrt{2}) \sqrt{2-\sqrt{2}}+2 \sqrt{2}}{8}\right]^{\frac{1}{2}} ; \\
& \left(\delta \sigma B_{1 *}\right)^{\frac{1}{\sigma}}=\left[\frac{(4+3 \sqrt{2}) \sqrt{2-\sqrt{2}}+2 \sqrt{2}}{8}\right]^{2} ; \\
& \rho^{*}=E^{*}+B_{2}^{*}=(40+30 \sqrt{2}) \sqrt{2-\sqrt{2}}+20 \sqrt{2} \text {; } \\
& \max \left\{\left(\delta \sigma B_{1 *}\right)^{\delta},\left(\delta \sigma B_{1 *}\right)^{\frac{1}{\sigma}}\right\}=\frac{(12+8 \sqrt{2}) \sqrt{2-\sqrt{2}}+7 \sqrt{2}+14}{32} \text {; }
\end{aligned}
$$


and $\rho^{*}>\max \left\{\left(\delta \sigma B_{1 *}\right)^{\delta},\left(\delta \sigma B_{1 *}\right)^{\frac{1}{\sigma}}\right\}$. So the condition (1.4) is satisfied. Moreover,

$$
\gamma(t)=\sum_{s=1}^{4} G(t, s)\left(-c_{0}\right)=-(4+3 \sqrt{2}) \sqrt{2-\sqrt{2}} \cdot c_{0}-2 \sqrt{2} c_{0},
$$

and so

$$
\gamma^{*}=\gamma_{*}=-(4+3 \sqrt{2}) \sqrt{2-\sqrt{2}} \cdot c_{0}-2 \sqrt{2} c_{0}<0 .
$$

Finally, since $c_{0} \in\left(0, \frac{3 \cdot[8 \sqrt{10}]^{-4 / 3}}{((4+3 \sqrt{2}) \sqrt{2-\sqrt{2}}+2 \sqrt{2})^{1 / 3}}\right)$, it follows that

$$
\gamma_{*} \geq-3\left[\frac{((4+3 \sqrt{2}) \sqrt{2-\sqrt{2}}+2 \sqrt{2})^{\frac{1}{2}}}{8 \sqrt{10}}\right]^{\frac{4}{3}}=\left[\frac{B_{1 *}}{\left(\rho^{*}\right)^{\sigma}} \delta \sigma\right]^{\frac{1}{1-\delta \sigma}}\left(1-\frac{1}{\delta \sigma}\right) .
$$

\section{Consequently, Theorem 1.3 yields that (4.2) has a positive solution. $\square$}

\section{Acknowledgements}

The authors are very grateful to the anonymous referees for their valuable suggestions. This work was supported by the NSFC (No. 11061030), NSFC (No. 11126296), the Fundamental Research Funds for the Gansu Universities.

\section{Authors' contributions}

RM completed the main study, carried out the results of this article and drafted the manuscript. YL checked the proofs and verified the calculation. All the authors read and approved the final manuscript.

\section{Competing interests}

The authors declare that they have no competing interests.

\section{Received: 28 March 2012 Accepted: 27 June 2012 Published: 27 June 2012}

\section{References}

1. Atici, FM, Guseinov, GS: Positive periodic solutions for nonlinear difference equations with periodic coefficients. J Math Anal Appl. 232, 166-182 (1999). doi:10.1006/jmaa.1998.6257

2. Atici, FM, Cabada, A: Existence and uniqueness results for discrete second-order periodic boundary value problems. Comput Math Appl. 45, 1417-1427 (2003). doi:10.1016/S0898-1221(03)00097-X

3. Atici, FM, Cabada, A, Otero-Espinar, V: Criteria for existence and nonexistence of positive solutions to a discrete periodic boundary value problem. J Diff Equ Appl. 9(9), 765-775 (2003). doi:10.1080/1023619021000053566

4. Ma, R, Ma, H: Positive solutions for nonlinear discrete periodic boundary value problems. J Appl Math Comput. 59, 136-141 (2010). doi:10.1016/j.camwa.2009.07.071

5. He, T, Xu, Y: Positive solutions for nonlinear discrete second-order boundary value problems with parameter dependence. J Math Anal Appl. 379(2), 627-636 (2011). doi:10.1016/j.jmaa.2011.01.047

6. $\mathrm{Ma}, \mathrm{R}, \mathrm{Lu}, \mathrm{Y}, \mathrm{Chen}, \mathrm{T}$ : Existence of one-signed solutions of discrete second-order periodic boundary value problems. Abstr Appl Anal 2012, 13 (2012). (Article ID 437912)

7. Lazer, AC, Solimini, S: On periodic solutions of nonlinear differential equations with singularities. Proc Am Math Soc. 99, 109-114 (1987). doi:10.1090/S0002-9939-1987-0866438-7

8. Gordon, WB: Conservative dynamical systems involving strong forces. Trans Am Math Soc. 204, 113-135 (1975)

9. Gordon, WB: A minimizing property of Keplerian orbits. Am J Math. 99, 961-971 (1977). doi:10.2307/2373993

10. Bonheure, D, Fabry, C, Smets, D: Periodic solutions of forced isochronous oscillators at resonance. Discret Contin Dyn Syst. 8(4), 907-930 (2002)

11. Fonda, A, Mansevich, R, Zanolin, F: Subharmonics solutions for some second order differential equations with singularities. SIAM J Math Anal. 24, 1294-1311 (1993). doi:10.1137/0524074

12. Jiang, D, Chu, J, Zhang, M: Multiplicity of positive periodic solutions to superlinear repulsive singular equations. J Diff Equ. 211(2), 282-302 (2005). doi:10.1016/j.jde.2004.10.031

13. del Pino, M, Manásevich, R, Montero, A: T-periodic solutions for some second order differential equations with singularities. Proc R Soc Edinburgh Sect A. 120(3-4), 231-243 (1992). doi:10.1017/S030821050003211X

14. Rachunková, I, Staněk, S, Tvrdý, M: Singularities and Laplacians in Boundary Value Problems for Nonlinear Ordinary Differential Equations, Handbook of Differential Equations (Ordinary Differential Equations). Elsevier, Amsterdam. 3 (2006)

15. Torres, PJ, Zhang, M: Twist periodic solutions of repulsive singular equations. Non-linear Anal. 56, 591-599 (2004) 
16. Rachunková, I, Tvrdý, M, Vrkoč, I: Existence of nonnegative and nonpositive solutions for second order periodic boundary value problems. J Diff Equ. 176, 445-469 (2001). doi:10.1006/jdeq.2000.3995

17. Torres, PJ: Existence of one-signed periodic solutions of some second order differential equations via a Krasnoselskii fixed point theorem. J Diff Equ. 190, 643-662 (2003). doi:10.1016/50022-0396(02)00152-3

18. Torres, PJ: Weak singularities may help periodic solutions to exist. J Diff Equ. 232, 277-284 (2007). doi:10.1016/j. jde.2006.08.006

19. Franco, D, Webb, JKL: Collisionless orbits of singular and nonsingular dynamical systems. Discret Contin Dyn Syst. 15, 747-757 (2006)

20. $\mathrm{Chu}, \mathrm{J}, \mathrm{Li}, \mathrm{M}$ : Positive periodic solutions of Hill's equations with singular nonlinear perturbations. Nonlinear Anal. 69, 276-286 (2008). doi:10.1016/j.na.2007.05.016

21. Agarwal, RP, O'Regan, D: Singular discrete boundary value problems. Appl Math Lett. 12, 127-131 (1999)

22. Agarwal, RP, Perera, K, O'Regan, D: Multiple positive solutions of singular and nonsingular discrete problems via variational methods. Nonlinear Anal. 58, 69-73 (2004). doi:10.1016/..na.2003.11.012

23. Lü, H, O'Regan, D, Agarwal, RP: Positive solution for singular discrete boundary value problem with sign-changing nonlinearities. J Appl Math Stoch Anal 2006, 1-14 (2006). (Article ID 46287)

24. Jiang, D, Pang, PYH, Agarwal, RP: Upper and lower solutions method and a superlinear singular discrete boundary value problem. Dyn Syst Appl. 16, 743-754 (2007)

doi:10.1186/1687-1847-2012-90

Cite this article as: Ma and Lu: Positive periodic solutions of second-order difference equations with weak singularities. Advances in Difference Equations 2012 2012:90.

\section{Submit your manuscript to a SpringerOpen ${ }^{\circ}$} journal and benefit from:

- Convenient online submission

- Rigorous peer review

- Immediate publication on acceptance

- Open access: articles freely available online

- High visibility within the field

- Retaining the copyright to your article

Submit your next manuscript at $\gg$ springeropen.com 